\title{
Complications following replantation of primary teeth: a case report
}

Mariana Oliveira Guimarães 1

(iD) https://orcid.org/0000-0002-4934-1055

Lorena Teixeira Melo Bomfim 2

(iD https://orcid.org/0000-0002-6297-2716

Paulo Antônio Martins-Júnior 3

(iD https://orcid.org/0000-0002-1575-5364

Fernanda Bartolomeo Freire-Maia 4

(iD) https://orcid.org/0000-0001-8083-9831
José Carlos Pettorossi Imparato 5

(iD https://orcid.org/0000-0002-1990-2851

Patrícia Maria Zarzar 6

iD https://orcid.org/0000-0002-6952-5767

\footnotetext{
1,3,4,6 Department of Child and Adolescent Oral Health. Faculty of Dentistry. Federal University of Minas Gerais. Rua Prof. Moacir Gomes de Freitas, 688. Pampulha. Belo Horizonte, MG, Brazil. CEP: 31.270-901. E-mail: marianaolig@hotmail.com

2 São Leopoldo Mandic University. Belo Horizonte, MG, Brazil.

5 Department of Pediatric Dentistry. School of dentistry. University of São Paulo. São Paulo, SP, Brazil.
}

\begin{abstract}
Introduction: the avulsion of primary teeth is a disturbing and unexpected event.

Description: this report describes the clinical case of a three-year-old child who suffered an avulsion and replantation of the primary upper central incisors at the site of the injury. The guardians sought treatment for the child at the Federal University of Minas Gerais after the replantation. Four months later, the child suffered a new trauma and the replanted teeth presented advanced mobility, root resorption and fistula. The clinical conduct was extraction and rehabilitation with a fixed esthetic maintainer.

Discussion: the literature describes two treatment options for avulsion of primary incisors: replantation and non-replantation. According to a recent systematic review, the difficulty in obtaining a consensus regarding the best clinical conduct is due, in part, to the scarcity of publications that present not only follow-ups with clinical success, but also with failures. The outcomes of replantation can be influenced by several factors. The time elapsed between replantation and splinting, and the new episode of trauma, negatively influenced the prognosis in the present case, leading to failure. Replantation of primary incisors is not yet evidence-based treatment. Therefore, this option must be chosen with caution and in ideal situations. It requires constant clinical and radiographic monitoring for evaluation of outcomes.
\end{abstract}

Key words Child, Tooth avulsion, Tooth replantation

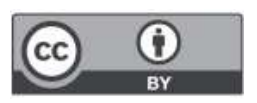




\section{Introduction}

Avulsion of primary teeth is described as the total displacement of the tooth from its alveolus ${ }^{1}$ and represents $7-13 \%$ of all types of traumatic dental injuries. 2,3 The upper incisors are the most frequent affected teeth due to their slight inclination towards vestibular and the directing of forces towards the palatal surface. ${ }^{4}$ Avulsion may also be associated with young age ${ }^{2}$ and child abuse. 5

The literature describes two treatment options with different success rates for handling cases of avulsion: replantation and non-replantation. ${ }^{6}$ The American Academy of Pediatric Dentistry does not recommend replantation of primary teeth due to risks of further damage to the permanent successor. ${ }^{1}$ On the other hand, studies have shown that factors such as maintenance of occlusal balance, speech, masticatory capacity, aesthetics and positive psychological effects encourage replantation in addition to the parents' wishes regarding the preservation of the tooth in the oral cavity. 7,8

According to a systematic review, 9 the difficulty in obtaining a consensus about the best clinical conduct to be adopted by pediatric dentists in cases of avulsion of primary teeth is due, in part, to the scarcity of publications that present not only followups with clinical success, but also with failures Furthermore, many researchers may feel discouraged to publish their results when there are negative clinical outcomes, ignoring the importance of their findings for the construction and consolidation of scientific evidence. ${ }^{9}$

Therefore, the aim of the present study was to describe the clinical case of a three-year-old child who suffered avulsion and replantation of the primary upper central incisors.

\section{Description}

A three-year-old child attended the specialized Trauma Center in Primary Dentition at Federal University of Minas Gerais after a dental trauma occurred three days before. The parents reported that the child had accidentally fallen and avulsed the primary upper central incisors (51 and 61). A dentist who was present at the accident site provided immediate care. The avulsed teeth were bathed in milk, the alveolus washed with filtered water, and then the teeth were replanted.

The time elapsed between the avulsion and the replantation was approximately 30 minutes. The dentist instructed the parents to seek a specialized pediatric dentistry service. No splinting was performed immediately after the replantation.

During our clinical examination the replanted teeth (51 and 61) were with moderate mobility and adjacent gingival tissue was lacerated. No occlusion interference was found. Radiographic examination revealed that the germs of the central permanent incisors were slightly rotated and in close relation with the roots of the primary central incisors. The dental follicle was expanded and no periapical lesion or signs of root resorption were detected (Figure 1A and $1 \mathrm{~B}$ ).

As the procedure had already been performed, with no signs and symptoms of infection and due to the parents' desire to maintain the teeth (considering the possible risks to permanent dentition), the clinical decision-making was the preservation of replanted teeth and continuous monitoring. A semirigid splinting was made $(0.5 \mathrm{~mm}$ steel wire and composite resin) (Figure 1C and 1D). The parents were instructed about child's oral hygiene and feeding.

The splinting should be used for 14 days, however, the patient failed to attend scheduled appointments. The child returned only 45 days after the initial appointment. The splinting was removed and a considerable reduction of teeth mobility was noted. A new radiograph was performed evidencing a beginning of external resorption, more evident in tooth 61. Pulp obliteration was observed in tooth 51 . No periapical lesion was identified (Figure 1E and 1F).

Four months after replantation, the child suffered a new trauma in the anterior region of the maxilla, with signals of subluxation in the upper central incisors. The child attended the Trauma Center and during the clinical examination, a moderate mobility of tooth 51 and an advanced mobility of tooth 61, associated with a fistula, were observed. Radiographic examination revealed advanced external root resorption and periapical lesions in both teeth (Figure 2A and 2B). As a clinical conduct, the teeth were extracted (Figure 2C and 2D) and the child was rehabilitated with a fixed aesthetic maintainer (Figure 2E and 2F). 


\section{Figure 1}

(A) Frontal view in occlusion (B) Initial $x$-ray (C,D) Semi-rigid splinting (E) Frontal view in occlusion after 45 days of semi-rigid splinting (F) $X$-ray after 45 days of semi-rigid splinting.
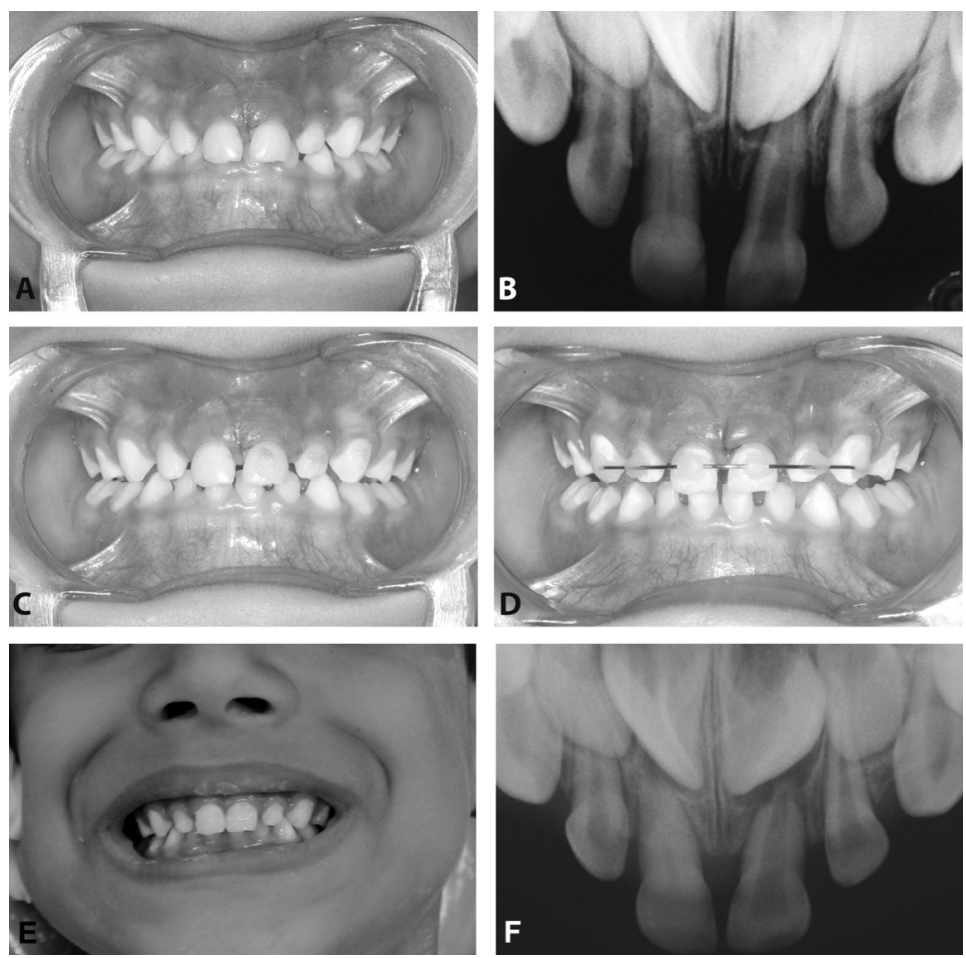

Figure 2

(A) Fistula associated with tooth 61 (B) X-ray confirming root resorption and periapical lesion. (C, D) Dental extraction $(E, F)$ Rehabilitation using aesthetic maintainer.
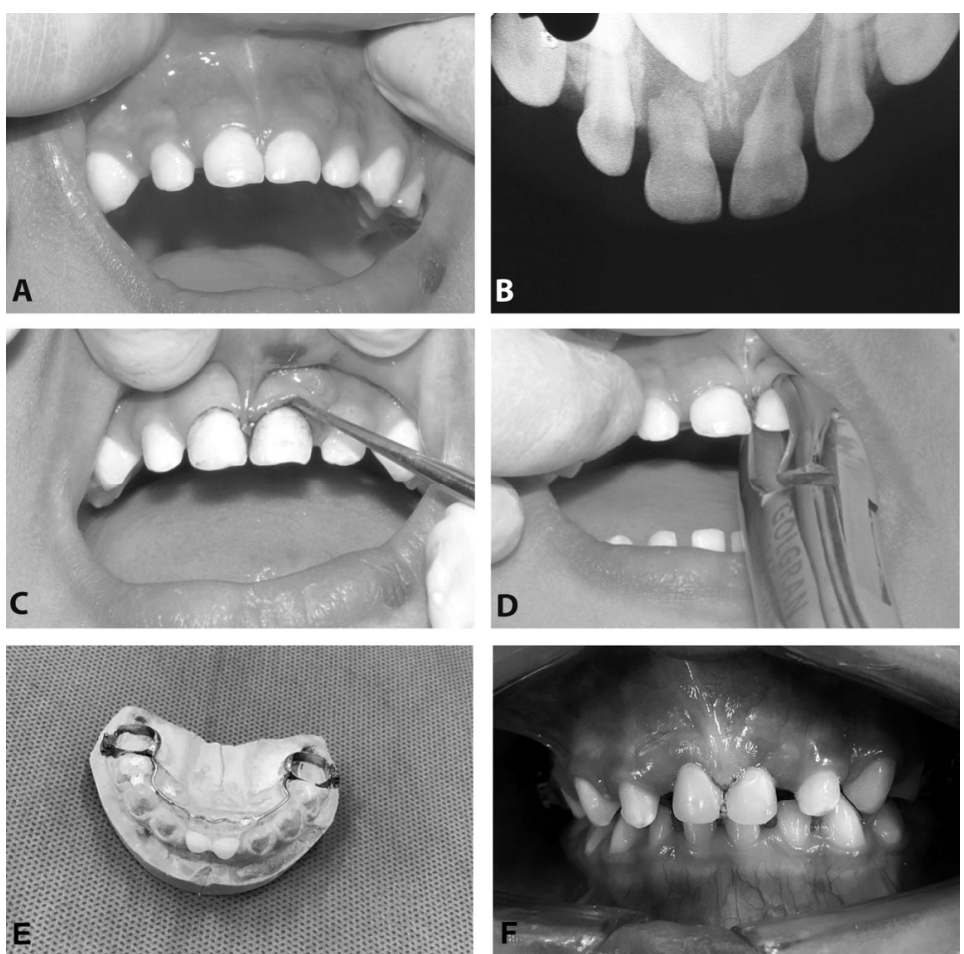


\section{Discussion}

The present study aimed to describe the clinical case of a three-year-old child who suffered avulsion and replantation of the primary upper central incisors, which lead to complications. Dental avulsion is considered a severe traumatic dental injury, commonly affecting children aged two to four years. 2,3 In the present case, the patient attended the clinic with the teeth already replanted. After four months of clinical and radiographic follow-up, the teeth were extracted due to severe external root resorption and a periapical lesion possible affecting the permanent successor.

Although not recommended, there is no consensus about the impossibility of replantation as an option of treatment. 9 Given favorable conditions, such as storage in solution, short extra-alveolar time, absence of rhizolysis, splinting and endodontic treatment, the prognosis may be favorable.7-9 In this sense, the first aspect to evaluate is the presence of root resorption, which contraindicates the replantation. ${ }^{8}$ In this present case, the replantation was not performed by the researchers, which makes it impossible to assure that there were no clinical signs of root resorption. Radiographic examination three days after trauma showed no evidence of root resorption in the replanted teeth.

Another aspect to consider is the time elapsed between avulsion and replantation. Some studies have shown that 30 minutes is the limit for the replantation since the cells of the periodontal ligament are still viable and the fluid clot can be removed by irrigation with sterile saline solution. 8,10 Because the first assistance was performed outside the clinical setting, the general dental practitioner did not have sterile saline solution to wash the alveolus, and did it with filtered water, but replantation was carried out in less than 30 minutes after avulsion.

The replanted teeth should receive semi-rigid splint, providing stabilization that allows physiological movement of the tooth, restoring the periodontal ligament and avoiding tooth aspiration. $8,10,11$ In this case, the splinting was performed 72 hours after the episode. When removed, both teeth presented slight mobility, evidencing a satisfactory recovery of the periodontium, although the late approach may have contributed to the failure of replantation.

Clinical and radiographic follow-up allows early identification of any signs and symptoms that indicate treatment failure. 7 The clinical and radiographic examination performed 45 days after the splinting removal revealed a beginning of external resorption. After four months, a fistula associated with tooth 61 and severe root resorption were found. Parent's report of a new trauma, added to the inflammatory process already installed, may have also contributed to the failure of replantation. The treatment outcome of replanted teeth can be influenced by several factors as it was presented in this paper. New traumatic dental injuries are one of the factors that may affect healing and consequently result in negatives outcomes. 12 It is important to highlight that children who have suffered previous dental trauma present a greater risk of suffering new episodes of dental trauma 13 and these can also affect long-term prognosis of replanted teeth. The teeth extraction following rehabilitation with esthetic maintainer was the treatment chosen.

The aesthetic rehabilitation of primary teeth has a positive psychological impact on the patient's selfesteem, since the absence of teeth causes difficulties for chewing, unpleasant esthetics and atypical lingual interposition. 14 In this case, the installation of an esthetic fixed maintainer was an appropriate treatment option, reestablishing both esthetics and function of the patient. The clinical and radiographic follow-up and maintenance of the esthetic maintainer should be done until the eruption of the permanent teeth, considering the possibility of damages to the successors at the time of the trauma or as a consequence of the replantation.

In the present case there was failure after replantation of avulsed primary teeth. Replantation of primary incisors is not yet an evidence-based treatment. Therefore, this option must be chosen with caution and in ideal situations. It requires constant clinical and radiographic monitoring for evaluation of the outcomes.

\section{Acknowledgement}

For the presentation of this case, the patient's parents assigned an informed consent form. This study was supported in part by Coordenação de Aperfeiçoamento de Pessoal de Nivel Superior (Coordination for the Improvement of Higher Education Personnel- Brazil (CAPES) and Conselho Nacional de Desenvolvimento Científico e Tecnológico (National Concil for Scientific and Technological Development (CNPq) .

\section{Author's contribution}

Guimarães MO, Bomfim LTM and Martins-Jr. PA: interpretation of data for the work, drafting, critical 
review and final approval of the version to be published. Freire-Maia FB, Imparato JCP and Zarzar PM: critical review and final approval of the version to be published.

\section{References}

1. Malmgren B, Andreasen JO, Flores MT, Robertson A, DiAngelis AJ, Andersson L, Cavalleri G, Cohenca N, Day P, Hicks ML, Malmgren O, Moule AJ, Onetto J, Tsukiboshi M. Guidelines for the management of traumatic dental injuries: 3. Injuries in the primary dentition. Pediatr Dent. 2017; 39: 420-8

2. Cunha RF, Pugliese DMC, Vieira AEM. Oral trauma in Brazilian patients aged 0-3 years. Dental traumatol 2001; 17: 229-36.

3. Harding AM, Camp JH. Traumatic injuries in the preschool child. Dent Clin North Am 1995; 39:817-835, 1995.

4. Mackie IC, Warren VN. Dental trauma: General aspects of management and trauma to the primary dentition. Dent update 1998; 15: 155-9.

5. Costacurta M, Benavoli D, Arcudi G, Docimo R. Oral and dental signs of child abuse and neglect. Oral Implantol. 2016; 8: 68-73.

6. Holan G. Replantation of avulsed primary incisors: a critical review of a controversial treatment. Dent Traumatol. 2012; 29: 178-84.

7. Zamom E, Kenny DJ. Replantation of avulsed primary incisors: a risck-benefit assessment. J Can Dent Assoc. 2001; 67: 368 .

8. Carvalho Rocha MJ, Cardoso M. Reimplantation of primary tooth case report. Dental traumatol. 2008; 24: 4-10.
9. Martins-Júnior PA, Franco FA, Barcelos RV, Marques LS, Ramos-Jorge ML. Replantation of avulsed primary teeth: a systematic review. Int J Paediatr Dent. 2014; 24: 77-83.

10. Christophersen P, Freund M, Harild L. Avulsion of primary teeth and sequelae on the permanent successors. Dent Traumatol. 2005; 21: 320-3.

11. Gatewood JC, Thornton JB. Successful replantation and splinting of a maxillary segment fracture in the primary dentition. Pediart Dent. 1995; 17: 124-6.

12. Soares Ade J, Gomes BP, Zaia AA, Ferraz CC, de SouzaFilho FJ. Relationship between clinical-radiographic evaluation and outcome of teeth replantation. Dent Traumatol. 2008; 24: 183-8.

13. Magno MB, Neves AB, Ferreira DM, Pithon MM, Maia LC. The relationship of previous dental trauma with new cases of dental trauma. A systematic review and metaanalysis. Dent Traumatol. 2019; 35: 3-14.

14. Khare V, Anand N, Vishal K, Ullal N. Fixed functional space maintainer: novel aesthetic approach for missing maxillary primary anterior teeth. BMJ Case Rep. 2013 : bcr2013009585.
Received on July 1, 2020

Final version presented on November 11, 2020

Approved on February 25, 2021 